\title{
Comparison of the Improved Coconut Hybrid CRIC65 with its Reciprocal Cross and the Parental Varieties for Reproductive Traits
}

\author{
S.A.C.N. Perera ${ }^{*}$, H.D.M.A.C. Dissanayaka and H.M.N.B. Herath
}

\begin{abstract}
Coconut is an important perennial tree crop which offers a multitude of uses. CRIC65 is the first recommended Sri Lankan hybrid coconut cultivar. Sri Lanka Green Dwarf and Sri Lanka tall are respectively, the maternal and paternal parents of CRIC65 and the hybrid is characterized by precocity, high nut yield and fruit component traits. Our objective in this study was to assess the comparative performance of reproductive traits of CRIC65 and its reciprocal hybrid, Sri Lanka Tall x Sri Lanka Green Dwarf with their parental cultivars. The annual nut yield and the fruit components were scored in sixty sample palms representing each cultivar. The field experiment of the two parents were planted in Kurunegala in a favourable site for coconut and the palms were 18 years old at the initiation of data recording. Time taken for flower initiation of the two hybrids and the data of the parental cultivars were extracted from previous records. The data of the two hybrids were analyzed by Student's t test. Reproductive traits, time taken for flowering and the annual nut yield were not significantly different, while fruit components, husked nut weight, split nut weight and kernel weight recorded significant differences on a few occasions but without identifying a better performer. The two hybrids were clearly different from their parents for the reproductive traits. In conclusion, we report the equal potential of the reciprocal cross (Sri Lanka Tall x Sri Lanka Green Dwarf) of
\end{abstract}

Genetics \& Plant Breeding Division, Coconut Research Institute, Lunuwila, Sri Lanka

*chandrikaperera2003@yahoo.com the main recommended hybrid coconut CRIC65 with respect to the most important reproductive parameters that define CRIC65 in favourable sites for coconut in Sri Lanka.

Keywords: Cocos nucifera (L.), Dwarf $\mathrm{x}$ Tall hybrids, Improved Cultivars, Reciprocal Crosses, Seed-nut production strategies

\section{INTRODUCTION}

Coconut (Cocos nucifera L.) is a tropical woody perennial plant belonging to the mono-species genus Cocos in the family Arecaceae. Coconut possess a diploid genome with 16 pairs $(2 n=2 x=32)$ of chromosomes. Globally, coconut has broadly been classified into two groups as 'tall' and 'dwarf' coconuts. Three varieties of coconuts have been identified in Sri Lanka as tall (typica), dwarf (nana) and intermediate (aurantiaca) (Liyanage, 1958).

Coconut is one of the major plantation crops in the tropics. Coconut palm offers a multitude of uses and thus has been termed as the "tree of life". It is socially and economically blended with the people in many of the coconut growing countries and has been identified as a crop having the potential for poverty alleviation in the rural areas of the coconut growing countries. 
Sri Lanka is the $4^{\text {th }}$ largest coconut producing country in the world. Total land area of cultivation under coconut is 395,000 ha (Anonymous, 2012a) which is the second largest extent under a single crop in the country. The coconut sector plays an important role in contributing to the national economy and ensuring food and nutrition security to a larger segment of the population. It accounts for about $1.7 \%$ of the Gross Domestic Production and contributes $2.74 \%$ of foreign exchange earning annually (Anonymous, 2012b).

The fruit (which is referred to as the 'nut') is the main yield of coconut. A national need of 3,000 million nuts is the estimated annual requirement of coconut to satisfy the demand on the consumers and the industry in Sri Lanka. Coconut breeding programmes conducted by the Coconut Research Institute of Sri Lanka (CRISL) aim at increasing the productivity in order to fulfil this demand. Hybrid coconuts of dwarf and tall coconuts have been proven to have a higher productivity compared to other cultivars (Dissanayaka et al., 2008; Perera et al., 2010).

The first hybrid coconut developed and recommended in Sri Lanka is CRIC65, which is a cross between the maternal parent Sri Lanka Green/Yellow dwarf and the paternal parent Sri Lanka Tall. CRIC65 has been recommended in the year 1965 and remains the main coconut hybrid in the country up-to-date. Seed-nuts of this coconut hybrid is mass produced by directed natural pollination at the isolated coconut seed garden at Ambakelle. The total annual production of seed-nuts of CRIC65 is less than 100,000 (unpublished records of CRISL) which is not sufficient to meet the much higher demand for this improved coconut hybrid. Expansion of the seed gardens are limited due to the extreme difficulty in finding suitable lands for the purpose. Accordingly, alternative strategies will be essential in the long run for the production of the planting materials of improved hybrid coconuts including CRIC65.

In the hybridization of tall and dwarf forms of coconut, the dwarf variety is used as the female parent for practical easiness of climbing the tree repeatedly for the process of emasculation or pollination. Even in the concept of directed natural pollination for mass production of hybrid seed-nuts it is essential to climb the tree for emasculation purposes. The phenomenon is common for CRIC65 in which the dwarf variety has been used as the maternal parent. Although there has been no evidence for the maternal inheritance in coconut so far, it is worthwhile to compare the performance of the genetically improved coconut cultivars with their reciprocal crosses.

The coconut hybrid CRIC65 is characterized by precocity (reduction of the duration of vegetative phase), high nut number $\quad 120$ nuts/palm/year) and fruit component parameters such as weights of fresh nut, dehusked nut and the kernel etc. In view of the information given above our objective in the current study was to assess the comparative performance of CRIC65 with 
its reciprocal cross for the important reproductive parameters affecting the performance and the productivity with a view of identifying alternative planting material.

\section{MATERIALS AND METHODS}

\section{Experimental Site}

The two coconut cultivars, CRIC65 (Sri Lanka Green Dwarf x Sri Lanka Tall) and its reciprocal cross (Sri Lanka Tall x Sri Lanka Green Dwarf) referred to as, GDT and TGD respectively were used in the study. The plants were at the age of 18 years at the initiation of data recording and were planted along with three other cultivars in a Randomized Complete Block Design. Each cultivar was represented by sixty palms for the recording of data.

\section{Reproductive Data Scored}

Yield and fruit component data were recorded over a period of six years from 2005 to 2010. Annual nut yield was recorded in each palm by recording the yield data in six picks per year at bi-monthly intervals.

Fruit component analysis was performed in a sample of sixty nuts per pick in each cultivar using a single nut from each sample palm and the fruit component analysis was repeated in each of the six picks in a given year. Recording of fruit component data was carried out in four steps by measuring the fruit weight (FNW) immediately after harvesting, followed by recording the weights of dehusked (HNW) and split nuts (SNW) and the kernel weights $(\mathrm{KW})$ in each nut sampled.

Secondary data on the time taken for flower initiation in the experimental palms were extracted from the records to assess the precocity of the two cultivars. Furthermore, secondary data of fifty individuals each of the parental varieties over a period of six years were extracted for comparison purposes.

\section{Data Analysis}

Student's t-test was performed in SAS V 8.0 to compare the scored parameters between two cultivars.

\section{RESULTS AND DISCUSSION}

\section{Flowering Time}

Two cultivars GDT and TGD recorded mean periods of 52 and 53 months respectively for the initiation of flowering from the date of field planting. Statistical analysis of the data did not reveal a significant difference between the two cultivars, with respect to the length of the vegetative phase or more importantly, attaining the reproductive stage of flowering and fruiting.

\section{Nut Yield}

No statistical difference was observed for the trait annual nut yield, in the two cultivars indicating the equal potential for yielding ability of the two reciprocal crosses (Figure 1). 


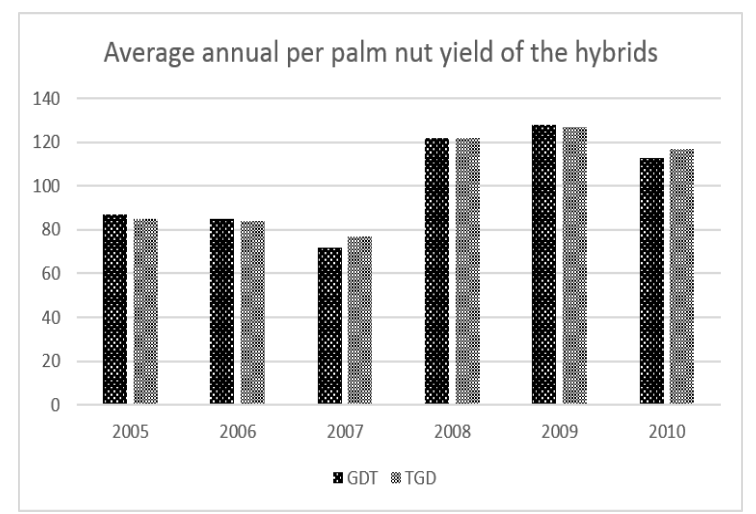

Figure 1: Average annual nut yield of the reciprocal crosses

\section{Fruit Component Analysis}

Fresh nut weight of the two cultivars was not significantly different between CRIC65 and its reciprocal cross in any of the years the data were recorded (Figure 2a). A statistically significant difference was observed for HNW in the year 2010 at $95 \%$ confidence interval with GDT recording a higher value compared to TGD (Figure 2b). Similarly, significant differences were identified for SNW in three years, but with the better performer being, GDT in 2007 and 2010 while TGD performed better than GDT in the year 2008 (Figure 2c). Weight of the kernel did not reveal any significant difference between two cultivars during the five initial years, yet GDT recorded significantly higher kernel weight than its reciprocal cross in the year 2010 (Figure 2d).

\section{Comparison of Reproductive Parameters of Reciprocal Crosses with Parental Varieties}

The reproductive data of the parental cultivars of the two hybrids (average values of fifty palms recorded over a period of six years) were extracted from previous records and are presented in table1. The parents recorded extreme values for flower initiation as 46 and 77 months respectively while the reciprocal crosses recorded statistically similar values of 52 and 53 months. All the rest of the parameters, (annual per palm nut yield, FNW, HNW. SNW and KW recorded the same pattern with the parents recording the two extreme values while the reciprocal hybrids GDT and TGD recorded statistically similar values. Both the hybrids have equally inherited precocity and higher nut yield from the parent GD and higher weights of different fruit components from the parent SLT. This resulted in equal performance for reproductive traits scored in the two reciprocal crosses while the parents were quite apart for the said traits.

\section{Proportional Weights of Fruit Components}

The coconut comprises of four components of the fruit; husk (HW), shell (SW), water (WW) and the kernel (KW). The value of each component of the two cultivars was derived by averaging across the years and the reciprocal crosses along with their parental varieties were compared as a percentage of the total average weight of the fruit. The two reciprocal cultivars evaluated and their parental varieties recorded more or less similar proportions of the four different components as shown in Figure 3. 


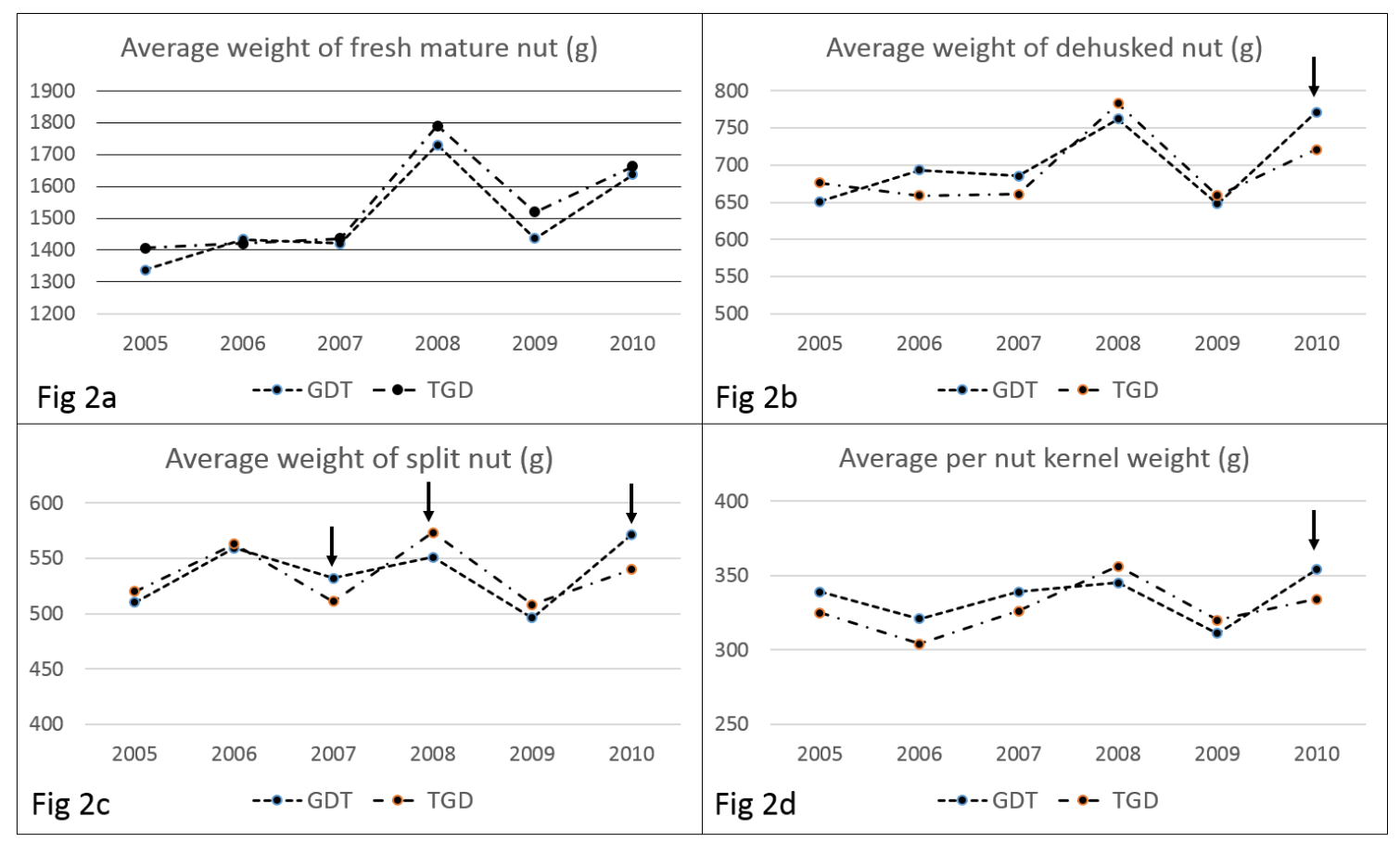

Note: Significantly different fruit components in each year are marked with an arrow

Figure 2: Average fruit components recorded by CRIC65 and its reciprocal cross over the period of 2005 to 2010 .

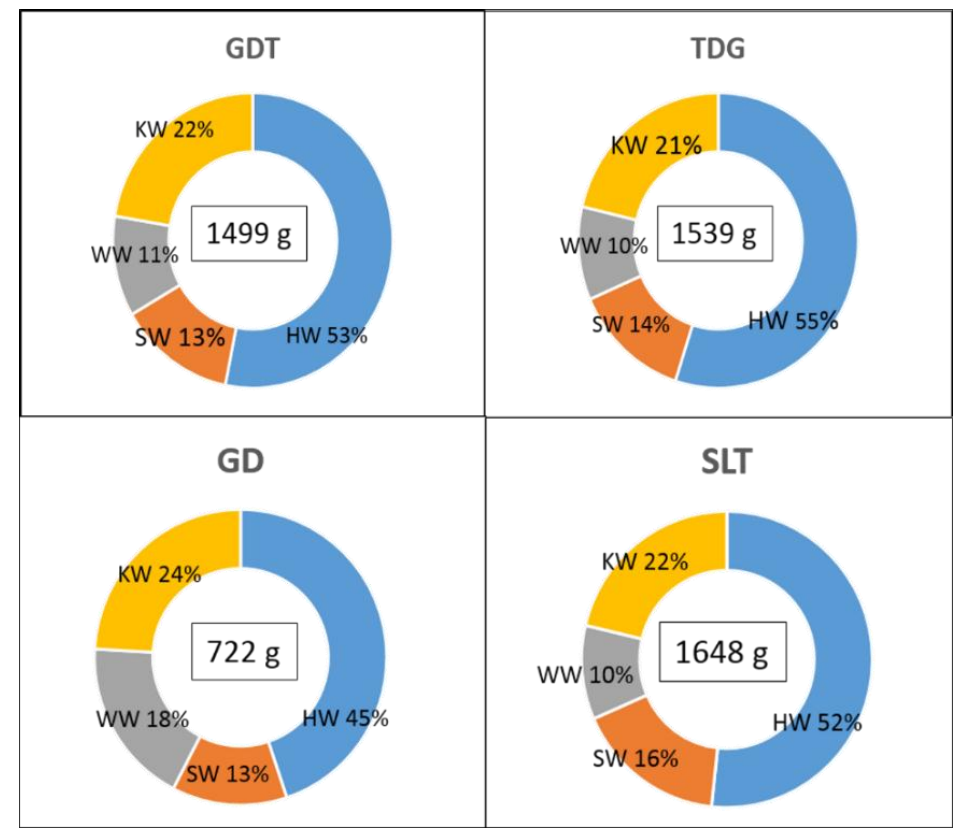

Figure 3: Each component of the fruit as a percentage of the total nut weight in CRIC65 and its reciprocal coconut hybrid (average weight of the fruit is given in the hollow of each diagram; $\mathrm{HW}=$ husk weight; $\mathrm{SW}=$ shell weight; $\mathrm{WW}=$ water weight and $\mathrm{KW}=$ kernel weight) 
Table 1: Average of reproductive parameters of reciprocal hybrids with their parental cultivars

\begin{tabular}{lllll}
\hline Variable & GDT & TGD & SLT & GD \\
\hline Flowering time (months) & 52 & 53 & 77 & 46 \\
Annual nut yield/palm & 101 & 102 & 70 & 116 \\
FNW (g) & 1499 & 1539 & 1648 & 722 \\
HNW (g) & 701 & 693 & 796 & 398 \\
SNW (g) & 536 & 535 & 627 & 351 \\
KW $(\mathrm{g})$ & 335 & 328 & 351 & 174 \\
\hline
\end{tabular}

\section{Implications of the Study}

The study revealed ample evidence to distinguish the two reciprocal hybrids from their parents while the two hybrids were similar in performance for the majority of the traits scored. The fruit components recorded significant differences between CRIC65 and its reciprocal cross in a few occasions but the better performer was not consistent. Accordingly, the current study does not provide any evidence for maternal inheritance for the traits scored in CRIC65 and its reciprocal cross.

There may be various weather parameters, which we have not evaluated in the current study affecting the performance in each year, accounting for the occasional significant differences of the fruit components. But in the macro cultivation scale, these variations will be common and such minor differences can be considered as trivial. Furthermore, despite the occasional significant differences fruit component analysis did not reveal a consistent better performer of the two cultivars while the flowering time and nut yield recorded similar performance. Literature is scarce on the comparative performance of reciprocal crosses of coconut hybrids in the world, preventing the comparison of the current findings.

However, the study provided substantial evidence for the equal reproductive performance of the two cultivars, in the given environment which is a favorable site for coconut cultivation, located within the coconut triangle. The seed garden capacity is not nearly adequate to meet the demand for the seedlings of the hybrid coconut CRIC65. As per the findings of this study, an alternative and equally potential cultivar would be the reciprocal cross of CRIC65 (Sri Lanka Tall x Sri Lanka Green Dwarf) to fulfil the demand and to be cultivated in favourable environments for coconut cultivation.

Sri Lanka Tall is the female parent of the coconut hybrid TGD and it is also the predominant coconut cultivar grown in commercial scale in Sri Lanka. Using selected tall in these plantations as the female parent, an alternative strategy for seed gardens is proposed for the mass production of TGD via medium scale artificial hand pollination of the selected tall coconut palms using processed pollen. Processed coconut pollen can be stored over a period of six months at $-200 \mathrm{C}$ maintaining the viability and the pollen processing can be carried out in a central facility. 


\section{CONCLUSION}

The improved coconut hybrid CRIC65 displays the equal potential with its reciprocal with respect to precocity and nut yield while they are clearly different with respect to the parents. In addition the fruit components of the two hybrids are comparable to a very high degree with varying performance occasionally. Yet this varying performance is not consistent to determine a better yielder with respect to fruit components. In conclusion, we confirm the equal potential of the reciprocal cross of the main coconut hybrid CRIC65 in relation to the most important reproductive parameters that define CRIC65 in favourable environments for coconuts in Sri Lanka.

\section{ACKNOWLEDGEMENT}

The authors wish to acknowledge the present and past staff of the Genetics and Plant Breeding Division of the Coconut Research Institute of Sri Lanka for the establishment and management of long term field experiments.

\section{REFERENCES}

Anonymous. (2012a). Plantation Sector Statistical Pocket Book.

Anonymous. (2012b). Annual Report of the Central Bank of Sri Lanka.

Dissanayaka, H.D.M.A.C., Perera, S.A.C.N., Fernando, W.B.S., Attanayake, R.B., Meegahakumbura, M.G.M.K. and Perera, L. (2008). Evaluation of the comparative performance of five commercial coconut cultivars under two different agro-ecological zones in Sri Lanka. In: Ninanayake, A. and Jayamanne, E. (Editors), Proceedings of the $2^{\text {nd }}$ Symposium on Plantation Crops Research, Colombo, Sri Lanka, pp 71-81

Liyanage, D.V. (1958). Varieties and forms of coconut palms grown in Ceylon. Ceylon Coconut Quarterly, 9 (1): 110.

Perera, S.A.C.N., Dissanayaka, H.D.M.A.C., Herath, H.M.N.B., Chandrasiri, S.A.S., Meegahakumbura, M.G.M.K. and Perera, L. (2010). Recently released coconut cultivars in Sri Lanka; A comparative evaluation. In: Dharmakeerthi, R.S. and Seneviratne, A.M.W.K. (Editors), Proceedings of the $3^{\text {rd }}$ Symposium on Plantation Crops Research, Colombo, Sri Lanka, pp 1. 